\title{
Investigation of avian rotavirus infections in broiler chicks from commercial flocks with different performance efficiency indexes
}

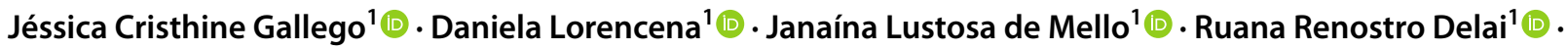

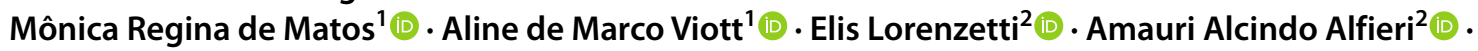 \\ Sergio Rodrigo Fernandes ${ }^{3}$ (i) . Elisabete Takiuchi ${ }^{1}$ (i)
}

Received: 1 October 2021 / Accepted: 18 February 2022 / Published online: 28 February 2022

(c) The Author(s), under exclusive licence to Springer Nature B.V. 2022

\begin{abstract}
The aim of this study was to investigate and compare the frequency of occurrence of avian rotavirus (AvRV) in poultry flocks according to its Performance Efficiency Index (PEI) scores. A total of 256 individual intestinal content samples of small sized-chicks (runts) with clinical signs of Runting Stunting Syndrome (RSS) and 24 clinically healthy chicks (control) were collected from twelve flocks in southern Brazil with different PEI scores: good $(n=4$, PEI mean $=365)$; moderate $(n=4$, PEI mean $=342)$ or poor $(n=4$, PEI mean $=319)$. Silver-stained polyacrylamide gel electrophoresis (ss-PAGE) was used to detect and identify the AvRV species followed by RT-PCR and sequencing of the partial VP6 gene for species confirmation. AvRV was detected in $83 \%$ (10/12) of the flocks and 23.4\% (60/256) of the chicks. The electrophoretic migration patterns of viral dsRNA segments were compatible with AvRV species A (AvRV- A), D (AvRV-D) and F (AvRV-F) in 9 (15\%), 18 (30\%), and $33(55 \%)$ of the positive chicks fecal samples, respectively. The AvRV species identified by ss-PAGE were confirmed by RT-PCR and partial sequence analysis of the VP6 gene. The AvRV detection rate was statistically higher $(p=0.007)$ in chicks from flocks with poor PEI when compared to those with good PEI. The occurrence of AvRV-D and AvRV-F was statistically higher in 7 to 9 days old chicks, while AvRV-A was detected only in 13 to 14 days old animals.
\end{abstract}

Keywords Rotavirus A · Rotavirus D · Rotavirus F · Ss-PAGE $\cdot$ Poultry flocks $\cdot$ Runting stunting syndrome

\section{Introduction}

Enteric diseases are one of the most important health problems that affect the poultry industry worldwide. Several pathogens have been identified as causes of intestinal tract infections of young chickens including those with signs of Runting Stunting Syndrome (RSS), also known as malabsorption syndrome. RSS is characterized by enteritis, anorexia, poor feathering, and severe weight loss, mostly within the first two weeks of age (Rebel et al. 2006). Commercial

Elisabete Takiuchi

e.takiuchi@ufpr.br

1 Departamento de Ciências Veterinárias, Universidade Federal Do Paraná - UFPR, Palotina, PR 85950-000, Brazil

2 Departamento de Medicina Veterinária Preventiva, Universidade Estadual de Londrina - UEL, Londrina, PR 86057-970, Brazil

3 Departamento de Zootecnia, Universidade Estadual de Londrina - UEL, Londrina, PR 86057-970, Brazil poultry flocks with RSS can determine significant economic losses mainly due to poor feed conversion and high discard rate which directly affect the flock's Performance Efficiency Index (PEI). The PEI is used by integrated poultry companies to evaluate flock performance and is equivalent to the European Broiler Index (EBI) that considers average daily gain (ADG), viability, and feed conversion ratio (FCR) (Marcu et al. 2013). Concerning enteric viruses, different agents have been identified in association with RSS including avian rotavirus (AvRV) (Devaney et al. 2016; Kang et al. 2012; Otto et al. 2006).

Rotaviruses are recognized causes of enteric diseases in a wide variety of avian and mammalian species. These viruses belong to the Reoviridae family and are classified into nine distinct species or groups (A to D and $\mathrm{F}$ to $\mathrm{J}$ ) based on the antigenicity and genetic characteristics of capsid protein VP6 (ICTV 2020; Matthijnssens et al. 2012). The rotavirus genome comprises 11 segments of double-stranded RNA (dsRNA) that migrate differently on silver-stained polyacrylamide gel electrophoresis (ss-PAGE), according to the 
rotavirus species. Birds can be infected by species of rotavirus species A (AvRV-A), D (AvRV-D), F (AvRV-F), and G (AvRV-G) (Todd and Mcnulty 1986).

Due to the multifactorial nature of RSS, is extremally difficult to establish cause-and-effect relationships conclusively on a field situation. However, to our best knowledge, epidemiological surveys in broiler chickens with signs of RSS in association with AvRV infections and PEI have never been conducted before and can be an interesting approach to evaluate the role of this virus on the flock's performance.

The aim of this study was to investigate and compare the frequency of occurrence of AvRV in intestinal content samples from broiler chicks with signs of RSS from commercial broiler flocks of a highly productive region of southern Brazil with different PEI scores.

\section{Materials and methods}

\section{Broiler flocks}

Twelve commercial broiler chicken flocks with stocking densities between 12 and 15.6 birds $/ \mathrm{m}^{2}$ were evaluated in 2015 . All the selected poultry farms were located in Paraná State, southern Brazil. This region is highly productive, which accounted for $24.8 \%$ of the national poultry production in 2015 (BRASIL 2016). Based on the PEI score (Marcu et al. 2013) the flocks were classified into three categories: good $(n=4$; mean PEI $=365)$, moderate $(n=4$; mean PEI $=342)$, or poor $(n=4$; mean PEI $=319)$.

\section{Animals and samples}

Twenty to twenty-two small-sized broiler chicks (runts) with clinical signs suggestive of RSS (lethargy, retarded growth, sticky droppings adhering to the cloaca and retarded feather development) and two normal-sized asymptomatic broiler chicks (control) were selected from each flock, performing a total of 280 mixed-sex broiler chicks of Cobb 500 strain. All chicks were between 7 and 14 days old and had feed and water ad libitum. The chicks were humanely euthanized by cervical dislocation and immediately submitted for necropsy to collect the enteric contents that were stored at $-20^{\circ} \mathrm{C}$ until analysis.

\section{Nucleic acid extraction and ss-PAGE}

The nucleic acid was extracted from $10 \%$ intestinal contents in Tris-calcium buffer $(10 \mathrm{mM}$ Tris- $\mathrm{HCl} ; 1.5 \mathrm{mM} \mathrm{CaCl} 2$; $\mathrm{pH}$ 7.3) using a combination of phenol/chloroform/isoamyl alcohol (25:24:1) and silica/guanidine thiocyanate according to Alfieri et al. (2006). DPEC water was used as negative control and a positive fecal sample for bovine rotavirus species A was included as the positive control. The extracted nucleic acid was submitted to 7.5\% PAGE followed by silver staining (Herring et al. 1982).

\section{RT-PCR and sequencing}

The AvRV ss-PAGE-positive fecal samples were submitted to RT-PCR with specific primers targeting the VP6 protein gene of the AvRV-A (Schumann et al. 2009), AvRV-D (Bezerra et al. 2012) and AvRV-F (Mascarenhas et al. 2016) for the species confirmation. The amplicons were purified by PureLink ${ }^{\circledR}$ Quick Gel Extraction Kit (Invitrogen, Carlsbad, CA, USA), quantified in a QubitTM Fluorometer, using QuantiTTM dsDNA BR Assay Kit (Invitrogen, Eugene, OR, USA). The sequencing reactions were performed in both directions with forward and reverse primers using an ABI3500 Genetic Analyzer sequencer and the BigDye ${ }^{\circledR}$ Terminator v3.1 Cycle Sequencing Kit (Applied Biosystems, Foster City, CA, USA). Sequence quality analysis was performed using Phred software and the consensus sequences were assembled using the CAP3 software (http://asparagin. cenargen.embrapa.br/phph/). Sequence similarity searches were performed using the Basic Local Alignment Search Tool (BLAST) software (http://blast.ncbi.nlm.nih.gov/). A nucleotide sequence of each rotavirus species was deposited in GenBank under accession numbers MZ935731 (AvRVD), MZ964147 (AvRV-A), and MZ964148 (AvRV-F).

\section{Statistical analysis}

The frequency data of AvRV and the different species detected in the broilers chicks were submitted to analysis of variance (ANOVA) concerning the flock PEI and the age groups ( 7 to 9 days and 13 to 14 days). Means for PEI groups were compared using the Tukey test, whereas the means for age groups were compared based on the F-test from ANOVA. A significance level of 0.05 was adopted for all analyses, which were performed in the Statistical Analysis System (SAS), v9.0.

\section{Results}

\section{AvRV detection by ss-PAGE and confirmation by RT-PCR and sequencing}

AvRV was detected in $83 \%(10 / 12)$ of evaluated broiler flocks and the identification of rotavirus species was obtained in 23.4\% (60/256) of the intestinal content samples analyzed by ss-PAGE. The electrophoretic migration profiles were characteristics of AvRV-A, AvRV-D, and AvRV-F in $9(15 \%), 18(30 \%)$, and $33(55 \%)$ of the positive fecal samples, respectively. The distribution of AvRV in the evaluated 


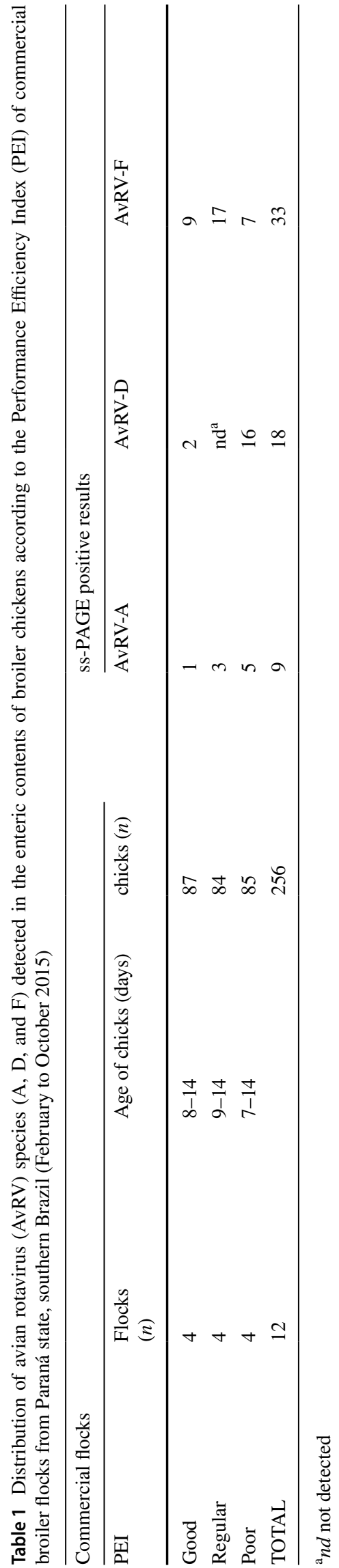

flocks is shown in Table 1. Figure 1 illustrates the electrophoretic profiles of AvRV dsRNA detected in this study. The RT-PCR and sequencing analysis confirmed the AvRV species previously identified from analysis of the electrophoretic profile by ss-PAGE.

A higher frequency of AvRV detection was observed in chicks from broiler flocks with poor PEI $(P=0.007)$ when compared to flocks with good PEI (Table 2). Regarding the distribution of AvRV species and the age group of affected chicks, the AvRV-A occurred only in broiler chicks at 13 to 14 days old $(P<0.001)$. In contrast, species $\mathrm{D}$ and $\mathrm{F}$ were observed significantly $(P=0.033$ and $P=0.050$, respectively) in chicks at 7 to 9 days of age.

AvRV was detected in only 2 (8.3\%) out of 24 asymptomatic control broiler chicks evaluated, with one sample positive for AvRV-A and one for AvRV-D, both from flocks with poor PEI scores.

\section{Discussion}

In the present survey, $23.4 \%$ of intestinal contents of broiler chicks evaluated were positive for AvRV by ss-PAGE. In studies that also used ss-PAGE as a diagnostic tool, the frequencies of occurrence of AvRV ranged from 0.86 to $64 \%$ in European countries and India (Karim et al. 2007; Otto et al. 2006, 2012). In Brazil, the frequencies ranged from 8.5 to $18 \%$ in the Minas Gerais and Paraná states (Alfieri et al. 1989; Tamehiro et al. 2003). AvRV dsRNA was detected in two control samples, however, the frequency found in asymptomatic broiler chicks was much lower than that in RSS broiler chicks, as already reported by other studies (Bezerra et al. 2012, 2014; McNulty and Reynolds 2008; Otto et al. 2006, 2012; Tamehiro et al. 2003). The wide variation in detection rates can be influenced by various factors including host susceptibility (age, lineage, and immunological condition), sample representativeness (number of samples and collection method) as well as laboratory analytical method (Karim et al. 2007; Otto et al. 2012; Pauly et al. 2017; Silva et al. 2013). The studies with the highest frequency rates sampled mostly symptomatic animals from flocks with RSS, individual samples, age group up to two weeks of age (considered the most susceptible) and RNA extraction protocols based on silica adsorption (Otto et al. 2006, 2012).

The highest detection rate of AvRV was observed in broilers chicks housed in poor PEI flocks with a statistical difference in comparison to the good PEI flocks. Several infectious risk factors have already been identified to negatively affect the flock's PEI in conventional broilers production systems, including coccidiosis and clostridiosis (Jones et al. 2018; Van Limbergen et al. 2020). However, to our knowledge, this is the first study in the world that evaluated the 
Fig. 1 Silver-stained polyacrylamide gel electrophoresis of the extracted nucleic acid of the enteric contents of runt chicks. The keys represent the number of genomic segments according to the electrophoretic migration profile of the AvRV species. Lanes 1 and 8: bovine RVA positive control ( $4: 2: 3: 2$ pattern); lanes 4 and 6: AvRV-A electropherogroup (5:1:3:2 pattern); lanes 2 and 7: AvRV-D electropherogroup (5:2:2:2 pattern); lanes 3 and 5: AvRV-F electropherogroup ( $4: 1: 2: 2: 2$ pattern)

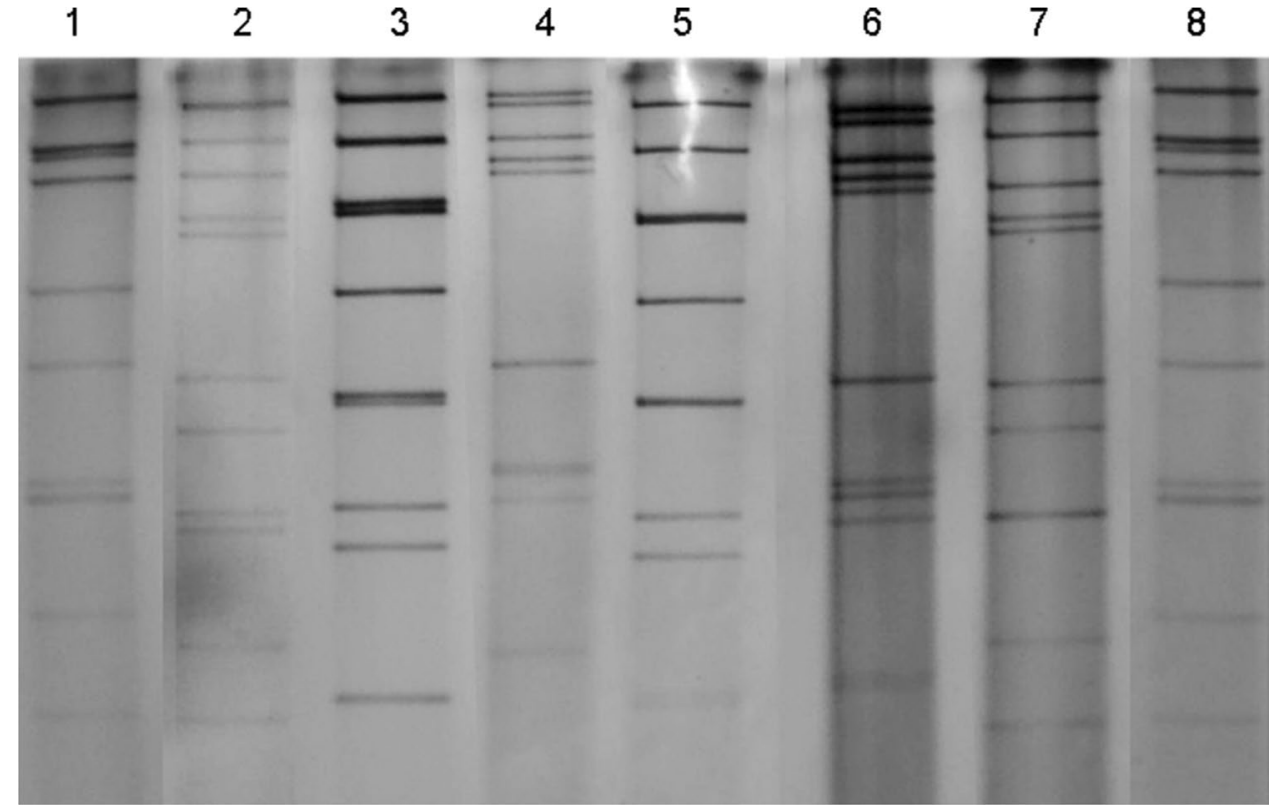

relationship between AvRV frequency and flock productive indexes. Silva et al. (2013) and Pauly et al. (2017) reported a higher occurrence of AvRV in flocks with high bird density, a non-infectious risk factor that magnifies poultry stress and facilitates the dissemination of AvRV.

AvRV-F was the most frequent (55\%), followed by AvRVD (30\%) and AvRV-A (15\%). In other studies, that also used the ss-PAGE as a diagnostic method, the detection rates for AvRV-A and AvRV-D ranged between 1.43 and 36\% (Alfieri et al. 1989; Bezerra et al. 2012, 2014; Karim et al. 2007; Otto et al. 2006; Tamehiro et al. 2003). With the use of more sensitive techniques such as RT-PCR, the detection rates varied from $16.1 \%$ to $58.8 \%$ and $39.2 \%$ to $53 \%$ for AvRV-A and AvRV-D, respectively (Bezerra et al. 2012; Otto et al. 2006, 2012).

The lowest concentration of rotavirus detected in human fecal samples by ss-PAGE was $10^{8}$ virions $/ \mathrm{mL}$ (Kohno et al. 2000). Due to the low sensitivity of the ss-PAGE technique

Table 2 Distribution of the avian rotavirus (AvRV) diagnostic frequency by silver-stained polyacrylamide gel electrophoresis (ss-PAGE) in broilers chicks, according to the Performance Efficiency Index (PEI) score of broiler flocks

\begin{tabular}{llll}
\hline PEI score & \multicolumn{3}{l}{ AvRV } \\
\cline { 2 - 4 } & Positive (\%) & Negative (\%) & TOTAL \\
\hline Good & $12(13.8)^{\mathrm{B}}$ & $75(86.2)$ & 87 \\
Regular & $20(23.8)^{\mathrm{AB}}$ & $64(76.2)$ & 84 \\
Poor & $28(32.9)^{\mathrm{A}}$ & $57(67.1)$ & 85 \\
TOTAL & $60(23.4)$ & $196(76.6)$ & 256 \\
\hline
\end{tabular}

Means with no common capital letters, in the same column, differed by Tukey's test $(\mathrm{p}<0.05)$
(Otto et al. 2006), positive results obtained in this assay indicate a high viral excretion at the time of sampling.

In contrast to the observation that the AvRV-F species occurs less frequently in broilers (Beserra and Gregori 2014; Bezerra et al. 2012; Otto et al. 2006) and is excreted in lower titers (Mascarenhas et al. 2016), in our study, the AvRV-F species was the most detected. Besides, in Brazil, AvRV-F had only been detected by RT-PCR assay with rates ranging from 9.4\% to 18.5\% (Beserra and Gregori 2014; Mascarenhas et al. 2016). This is the first report to identify AvRV-F by electrophoretic profile on ss-PAGE in Brazil. The individual collection of intestinal contents may be one of the explanations for the higher frequency of AvRV-F reported in our study. Beserra and Gregori (2014) and Mascarenhas et al. (2016) evaluated pools of fecal samples from different individuals. In this type of sampling, a positive fecal sample may become undetectable by the ss-PAGE technique after reducing the viral concentration by mixing with negative fecal samples. Meanwhile, AvRV-D was not detected in flocks with regular PEI score. Among the possible explanations for this are the absence of virus circulation in the flocks or the sampled birds would be with low viral load at the time of collection.

Considering the age range of the broilers chicks, several reports have indicated that the highest occurrence of AvRV is in the first two weeks of age (Decaesstecker et al. 1988; Mascarenhas et al. 2016; Tamehiro et al. 2003). Although our sampling represents a narrow range of age (7 to 14 days), the occurrence of AvRV-D and AvRV-F was statistically higher in the 7 to 9 days old age group, while AvRV-A was detected only in chicks between 13 to 14 days old. The non-detection of AvRV-A in broilers from 7 to 9 days of age corroborates 
the finding by McNulty et al. (1983) in a longitudinal survey, where AvRV-A was not detected in broilers younger than 14 days old.

Despite much research have been conducted to elucidate the etiology of RSS, it remains unclear. A large and growing number of viral pathogens and even a combination of multiple viruses have been associated with RSS in broiler chickens, such as reovirus (Songserm et al. 2002), astrovirus (Kang et al. 2012), rotavirus (Otto et al. 2012), calicivirus (Devaney et al. 2016), coronavirus (Hauck et al. 2016), parvovirus (Zsak et al. 2013), birnavirus (Noiva et al. 2015), and gallivirus (Oliveira et al. 2021). Because we did not investigate other pathogens, our findings are not sufficient to establish a causal relationship between AvRV and RSS. Due to the wide spectrum of possible etiologies, the differential diagnosis becomes quite difficult and complex, especially in field situations. In addition to being a time-consuming and very costly process, it is necessary to have well standardized protocols for the accurate detection of different pathogens. Furthermore, the interpretation of results may be challenging since many of these viruses were also detected in clinically healthy animals (Lima et al. 2019). In conclusion, the rate of AvRV detection in intestinal content samples of broiler chicks was high, mainly in flocks with poor PEI. The significant detection of AvRV in chicks with RSS does not constitute an independent indicator for the occurrence of RSS and poor performance of flocks. However, our results suggest that future studies with multivariable modelling should include the AvRV in order to determine whether rotaviruses are potential contributors to the RSS pathogenesis as well as whether it constitutes an important risk factor that threatens the flock's performance under natural field conditions.

Acknowledgements The authors would like to thank the Brazilian Federal Agency for Support and Evaluation of Graduate Education (CAPES/MEC) for granting a scholarship to the first author.

Authors' contributions This study was conceptualized by Jessica Cristhine Gallego and Elisabete Takiuchi; Jessica Cristhine Gallego, Daniela Lorencena, Janaína Lustosa de Mello, Ruana Renostro Delai, Mônica Regina de Matos, Aline de Marco Viott and Sergio Rodrigo Fernandes conducted experimental study; Jessica Cristhine Gallego, Elis Lorenzetti, Amauri Alcindo Alfieri and Elisabete Takiuchi performed sequence analysis; Jessica Cristhine Gallego and Elisabete Takiuchi written original draft preparation; Jessica Cristhine Gallego, Elisabete Takiuchi, Aline de Marco Viott, Sergio Rodrigo Fernandes, Elis Lorenzetti and Amauri Alcindo Alfieri written review and editing. All authors read and approved the final manuscript.

Funding This work was supported by Universidade Federal do ParanáUFPR, Universidade Estadual de Londrina-UEL and CAPES/MEC. Coordenação de Aperfeiçoamento de Pessoal de Nível Superior

Availability of data and material The raw data that support the findings of this study are available on request from the corresponding author.

Code availability Not applicable.

\section{Declarations}

Ethics approval The entire experimental procedure is following the ethical principles adopted by the local Ethics Commission from the Universidade Federal do Paraná under protocol number 15/2014.

Consent to participate All the authors consent to participate in publication.

Consent for publication All the authors consent to publish the manuscript.

Conflicts of interest The authors declare that they have no conflict of interest.

\section{References}

Alfieri AF, Resende M, Resende JS, Alfieri AA (1989) Atypical rotavirus infections among broiler chickens in Brazil. Arq Bras Med Vet Zootec 41:81-82

Alfieri AA, Parazzi ME, Takiuchi E, Médici KC, Alfieri AF (2006) Frequency of group A rotavirus in diarrhoeic calves in Brazilian cattle herds, 1998-2002. Trop Anim Health Prod. https://doi.org/ 10.1007/s11250-006-4349-9

Beserra LAR, Gregori F (2014) Description of rotavirus F in broilers from Brazilian poultry farms. Avian Dis 58:458-461. https://doi. org/10.1637/10747-121613-ResNote.1

Bezerra DAM, da Silva RR, Kaiano JH, de Souza OD, Gabbay YB, Linhares AC, Mascarenhas JD (2014) Detection, epidemiology and characterization of VP6 and VP7 genes of group D rotavirus in broiler chickens. Avian Pathol 43:238-243. https://doi.org/ 10.1080/03079457.2014.913097

Bezerra DAM, da Silva RR, Kaiano JH, Silvestre RV, de Souza OD, Linhares AC, Gabbay YB, Mascarenhas JD (2012) Detection of avian group $\mathrm{D}$ rotavirus using the polymerase chain reaction for the VP6 gene. J Virol Methods 185:189-192. https://doi.org/10. 1016/j.jviromet.2012.07.017

BRASIL (2016) Estatística da produção pecuária Municipal. Indicadores do Instituto Brasileiro de Geografia e Estatística-IBGE. Municipal Livestock Production 2015. https://biblioteca.ibge. gov.br/visualizacao/periodicos/84/ppm_2015_v43_br.pdf. Acessed 22 May 2020

Decaesstecker M, Charlier G, Meulemans G (1988) Epidemiological study of enteric viruses in broiler chickens: comparison of tissue culture and direct electron microscopy. Avian Pathol 17:477-486

Devaney R, Trudgett J, Trudgett A, Meharg C, Smyth V (2016) A metagenomic comparison of endemic viruses from broiler chickens with runting-stunting syndrome and from normal birds. Avian Pathol 45:619-629. https://doi.org/10.1080/03079457.2016.1193123

Hauck R, Gallardo RA, Woolcock PR, Shivaprasad HL (2016) A Coronavirus Associated with Runting Stunting Syndrome in Broiler Chickens. Avian Dis 60:528-534. https://doi.org/10.1637/ 11353-122215-Case

Herring AJ, Inglis NF, Ojeh CK, Snodgrass DR, Menzies JD (1982) Rapid diagnosis of rotavirus infection by direct detection of viral nucleic acid in silver-stained polyacrylamide gels. J Clin Microbiol 16:473-477

ICTV (2020) International Committee on Taxonomy of Viruses - Virus Taxonomy: 2020 Release. https://talk.ictvonline.org/taxonomy/. Accessed 25 January 2021

Jones PJ, Niemi J, Christensen JP, Tranter RB, Bennet RM (2018) A review of the financial impact of production diseases in poultry 
production systems. Anim Prod Sci 59:1585-1597. https://doi. org/10.1071/AN18281

Kang KI, El-Gazzar M, Sellers HS, Dorea F, Williams SM, Kim T, Collett S, Mundt E (2012) Investigation into the aetiology of runting and stunting syndrome in chickens. Avian Pathol 41:41-50. https://doi.org/10.1080/03079457.2011.632402

Karim M, Rume F, Alam M, Ahmed M (2007) Molecular epidemiologic study on avian rotavirus prevailing in Bangladesh. Bangladesh J Vet Med 5:43-48. https://doi.org/10.3329/bjvm.v5i1.1308

Kohno H, Akihara S, Nishio O, Ushijima H (2000) Development of a simple and rapid latex test for rotavirus in stool samples. Pediatr Int 42:395-400. https://doi.org/10.1046/j.1442-200x.2000. 01252.x

Lima DA, Cibulski SP, Tochetto C, Varela A, Finkler F, Teixeira TF, Loiko MR, Cerva C, Junqueira DM, Mayer FQ, Roehe PM (2019) The intestinal virome of malabsorption syndrome-affected and unaffected broilers through shotgun metagenomics. Virus Res 261:9-20. https://doi.org/10.1016/j.virusres.2018.12.005

Marcu A, Vacaru-Opris I, Dumitrescu G, Ciochina LP, Marcu A, Nicula M, Pet I, Dronca D, Kelciov B, Maris C (2013) The Influence of Genetics on Economic Efficiency of Broiler Chickens Growth. J Anim Sci Biotechnol 46:339-346

Mascarenhas JD, Bezerra DA, Silva RR, Silva MJ, Junior EC, Soares LS (2016) Detection of the VP6 gene of group F and G rotaviruses in broiler chicken fecal samples from the Amazon region of Brazil. Arch Virol 161:2263-2268. https://doi.org/10.1007/ s00705-016-2882-z

Matthijnssens J, Otto PH, Ciarlet M, Desselberger U, Van Ranst M, Johne R (2012) VP6-sequence-based cutoff values as a criterion for rotavirus species demarcation. Arch Virol 157:1177-1182. https://doi.org/10.1007/s00705-012-1273-3

McNulty MS, Reynolds DL (2008) Viral Enteric Infections-Rotavirus Infections. In: Saif YM, Fadly AM, Glisson JR, Mcdougald LR, Nolan LK, Swayne DE (Eds) Diseases of Poultry 12rd edn USA, pp329-350

McNulty MS, Allan GM, McCracken RM (1983) Experimental infection of chickens with rotaviruses: clinical and virological findings. Avian Pathol 12:45-54

Noiva R, Guy JS, Hauck R, Shivaprasad HL (2015) Runting stunting syndrome associated with transmissible viral proventriculitis in broiler chickens. Avian Dis 59:384-387. https://doi.org/10.1637/ 11061-031115-Case.1

Oliveira LB, Stanton JB, Zhang J (2021) Runting and stunting syndrome in broiler chickens: histopathology and association with a novel picornavirus. Vet Pathol 58:123-135. https://doi.org/10. 1177/0300985820969971

Otto P, Liebler-Tenorio EM, Elschner M, Reetz J, Lohren U, Diller R (2006) Detection of rotaviruses and intestinal lesions in broiler chicks from flocks with runting and stunting syndrome (RSS). Avian Dis 50:411-418. https://doi.org/10.1637/7511-020106R.1

Otto PH, Ahmed MU, Hotzel H, Machnowska P, Reetz J, Roth B, Trojnar E, Johne R (2012) Detection of avian rotaviruses of groups A, D, $\mathrm{F}$ and $\mathrm{G}$ in diseased chickens and turkeys from Europe and Bangladesh. Vet Microbiol 156:8-15. https://doi.org/10.1016/j.vetmic. 2011.10.001

Pauly M, Oni OO, Sausy A, Owoade AA, Adeyefa CAO, Muller CP, Hubschen JM, Snoeck CJ (2017) Molecular epidemiology of avian rotaviruses group A and D shed by different bird species in Nigeria. J Virol 14:111. https://doi.org/10.1186/s12985-017-0778-5

Rebel JMJ, Balk FRM, Post J, Van Hemert S, Zekarias B, Stockhofe N (2006) Malabsorption syndrome in broilers. Worlds Poult Sci J 62:17-29. https://doi.org/10.1079/WPS200481

Schumann T, Hotzel H, Otto P, Johne R (2009) Evidence of interspecies transmission and reassortment among avian group A rotaviruses. Virology 386:334-343. https://doi.org/10.1016/j.virol. 2009.01.040

Silva RR, Bezerra DAM, Kaiano JHL, Manno MC, Oliveira DS, Sagica FES, Gabbay YB, Chaves SOC, Silva ALC, Alfieri AA, Mascarenhas JDAP (2013) Molecular epidemiology of avian rotavirus in fecal samples of broiler chickens in Amazon Region, Brazil, from August 2008 to May 2011. Rev Pan-Amaz Saude 4:55-62. https://doi.org/10.5123/ S2176-62232013000200007

Songserm T, Zekarias B, van Roozelaar DJ, Kok RS, Pol JM, Pijpers AA, ter Huurne AA (2002) Experimental reproduction of malabsorption syndrome with different combinations of reovirus, Escherichia coli, and treated homogenates obtained from broilers. Avian Dis 46:87-94. https://doi.org/10.1637/0005-2086(2002) 046[0087:EROMSW]2.0.CO;2

Tamehiro CY, Alfieri AF, Médici C, Alfieri AA (2003) Segmented double-stranded genomic RNA viruses in fecal samples from broiler chicken. Braz J Microbiol 34:344-348. https://doi.org/10. 1590/S1517-83822003000400013

Todd D, McNulty MS (1986) Electrophoretic variation of avian rotavirus RNA in polyacrylamide gels. Avian Pathol 15:149-159

Van Limbergen T, Sarrazin S, Chantziaras I, Dewulf J, Ducatelle R, Kyriazakis I, McMullin P, Méndez J, Niemi JK, Papasolomontos S, Szeleszczuk P, Erum JV, Maes D (2020) Risk factors for poor health and performance in European broiler production systems. BMC Vet Res 16:287. https://doi.org/10.1186/s12917-020-02484-3

Zsak L, Cha RM, Day JM (2013) Chicken parvovirus-induced runting-stunting syndrome in young broilers. Avian Dis 57:123-127. https://doi.org/10.1637/10371-091212-ResNote.1

Publisher's Note Springer Nature remains neutral with regard to jurisdictional claims in published maps and institutional affiliations. 